\title{
Bioactive Lignans from a Cultivar of
}

\section{Helianthus annuus}

FRANCISCO A. MACÍAS,* ADRIANA LÓPEZ, ROSA M. VARELA, ASCENSIÓN TORRES and JOSÉ M. G. MOLINILLO

Grupo de Alelopatía, Departamento de Química Orgánica, Facultad de Ciencias,
Universidad de Cádiz, Apdo 40, 11510- Puerto Real, Cádiz, Spain.
(supporting information)

Bioactive lignans from cultivar sunflower

* To whom correspondence should be addressed. Tel.:+34(956)016370

Fax:+34(956)016193. E-mail: famacias@uca.es. 
Structural types and QSAR properties calculated.

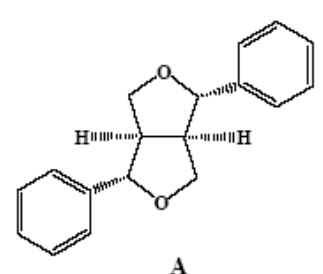

A

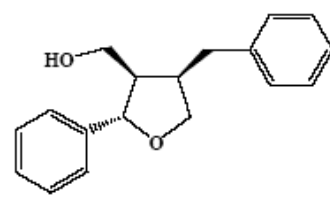

B<smiles>OCCCc1ccccc1</smiles>

C

\begin{tabular}{l|rrrr} 
Compound & $\mathbf{1}$ & $\mathbf{2}$ & \multicolumn{3}{c}{$\mathbf{3}$} & \multicolumn{1}{c}{$\mathbf{4}$} \\
\hline Number of hydrogen bond acceptors & 6 & 7 & 8 & 11 \\
Number of hydrogen bond donors & 2 & 2 & 2 & 4 \\
Molecular weight & 358 & 388 & 418 & 584 \\
IA logP & 1.9 & 1.64 & 3.29 & 3.15 \\
LogKow & 2.02 & 1.84 & 1.67 & 1.55 \\
C logP & 1.31 & 1.093 & 0.877 & 0.81 \\
IA log W & -3.23 & -3.06 & -4.02 & -4.26 \\
Number of Lipinski's rule & 4 & 4 & 4 & 2
\end{tabular}

\begin{tabular}{l|rr} 
Compound & $\mathbf{5}$ & \multicolumn{1}{c}{$\mathbf{7}$} \\
\hline Number of hydrogen bond acceptors & 6 & 7 \\
Number of hydrogen bond donors & 3 & 4 \\
Molecular weight & 360 & 376 \\
IA logP & 2.02 & 1.73 \\
LogKow & 2.34 & 1.21 \\
C logP & 1.426 & -0.331 \\
IA log W & -3.11 & -1.19 \\
Number of Lipinski's rule & 4 & 4
\end{tabular}

\begin{tabular}{l|rrr} 
Compound & \multicolumn{1}{|c}{} & \multicolumn{1}{c}{10} & \multicolumn{1}{c}{11} \\
\hline Number of hydrogen bond acceptors & 6 & 4 & 7 \\
Number of hydrogen bond donors & 3 & 2 & 4 \\
Molecular weight & 360 & 211 & 378 \\
IA logP & 2.02 & 1.21 & 0.79 \\
LogKow & 2.99 & 1.23 & 1.07 \\
C logP & 1.635 & 0.677 & 0.752 \\
IA log W & -3.11 & -1.49 & -2.26 \\
Number of Lipinski's rule & 4 & 4 & 4
\end{tabular}

